\title{
Integración de la bicicleta en el sistema de transporte público de la Comunidad de Madrid
}

\author{
Miguel Ángel Delgado Ruiz, Consorcio Regional de Transportes de Madrid. Luis \\ Criado Fernández, Consorcio Regional de Transportes de Madrid. Javier Rodríguez \\ Ranz, Consorcio Regional de Transportes de Madrid. Javier Moya Porras, Consorcio \\ Regional de Transportes de Madrid.
}

La integración de la bicicleta como parte del sistema de transporte público es un desafío y, a la vez, una oportunidad histórica para favorecer un modelo de transporte más sostenible. La bicicleta ha de ser tomada como aliada y complementaria a otros modos de transporte en una estrategia global de movilidad. En el caso de la Comunidad de Madrid, desde hace muchos años no ha existido una gran tradición de ciclismo urbano aunque el ciclismo deportivo sí que ha mantenido una amplia base social cimentada sobre todo en el boom de la bicicleta de montaña. Una serie de prejuicios, como la célebre afirmación de que "Madrid no es una ciudad para la bicicleta" están siendo afortunadamente superados y no hay más que salir a nuestras calles para constatarlo. Tanto en la capital como en el resto de la región madrileña, la visibilidad de la bicicleta es cada día mayor.

\section{Causas del aumento de la visibilidad de la bicicleta.}

El aumento de la presencia de la bicicleta en la Comunidad de Madrid se debe a unos factores que son comunes para otras ciudades de nuestra geografía, si bien es cierto que en el caso de nuestra región se parte de unos porcentajes de movilidad ciclista verdaderamente bajos, lo que le da mayor valor, si cabe, a esta incipiente movilidad a dos ruedas.

El papel de la Bicicleta Pública. El primer factor, aunque no necesariamente el más importante, es la presencia de sistemas de Bicicleta Pública (BP) en varios municipios de la Comunidad de Madrid. Estos sistemas, con mayor o menor éxito, han dado visibilidad a la bicicleta en estas ciudades del área metropolitana. A pesar de que hay municipios con BP desde hace varios años como Leganés, Getafe o Majadahonda, ha sido la puesta en marcha de BiciMad (denominación de la BP en Madrid capital) la que ha puesto, para bien o para mal, a la bicicleta en el foco mediático.

El fomento de los hábitos saludables. El segundo factor que justifica la mayor presencia de la bicicleta en la ciudad es la creación de una conciencia social de respeto al medioambiente y de fomento de hábitos saludables. En este sentido la promoción de la movilidad a pie o en bicicleta -los llamados modos blandos- es una de las estrategias básicas de los defensores de la sostenibilidad y de un nuevo modelo de ciudad donde el vehículo privado tenga limitaciones de acceso al centro urbano frente al transporte público, el peatón o la bicicleta.

La bicicleta como fenómeno cultural. Por último, y tampoco por ello menos relevante, hay que valorar que la bicicleta ha trasmutado desde su percepción de simple "artefacto" de uso deportivo a objeto de culto, epicentro de una moda que hace que la bicicleta tenga gran visibilidad en nuestras calles como parte de un movimiento que valora la estética "vintage", incluso en el modo de vestir de los ciclistas. En términos económicos el "boom" de la bicicleta está reportando un notable crecimiento en este 
sector no solo en el segmento comercial sino también en la distribución y fabricación de bicicletas y accesorios, con lo que se cierra un circulo virtuoso.

\section{La bicicleta en las políticas de transporte público.}

Abordar la integración de la bicicleta como un nuevo eslabón en la cadena modal exige de las instituciones públicas una visión transversal de la movilidad ciclista considerando todas y cada una de sus problemáticas así como las posibles soluciones a aplicar. El papel de una Autoridad de Transporte, como es el caso del Consorcio Regional de Transportes de Madrid (CRTM), debe ser el análisis de la problemática y necesidades del ciclista como potencial usuario del transporte público, conocer cuales son sus demandas e intentar satisfacer sus necesidades. En este sentido, y al margen de los estudios técnicos, es fundamental el diálogo que puede establecerse.

\section{Líneas de trabajo a favor de la bicicleta emprendidas desde el CRTM.}

Se han abierto cinco grandes líneas de trabajo, que incluyen aspectos tan interesantes como la red de itinerarios ciclistas, los estacionamientos de bicis, la modificación normativa de la bici a bordo del Transporte Público, las nuevas tecnologías de billetica y aspectos más relacionados con la bici y el ocio.

\section{-Política de infraestructuras y vías ciclistas así como su conexión a estaciones e intercambiadores de transporte, mejorando el acceso en bici a los mismos.}

Desde el CRTM se ha comenzado a elaborar un inventario de actuaciones focalizado en la definición y mejora de itinerarios seguros para bicicletas en los encaminamientos a estaciones e intercambiadores de transporte. Esta relación de "puntos de bloqueo" en la continuidad de rutas de media y larga distancia sirve para coordinar actuaciones y competencias de diversas administraciones que comparten responsabilidades en la resolución de estas conexiones. En este sentido, el número de instituciones implicadas es muy nutrido e incluye desde municipios colindantes que han de acordar como conectar los carriles bici de sus respectivas ciudades a los responsables, pasando por gestores institucionales de terrenos por donde se puede hacer una conexión ciclista (Ministerio de Defensa, ADIF, etc.).

Lograr mejorar la conectividad de un itinerario ciclista requiere de soluciones imaginativas que, en la mayoría de los casos, no son tampoco costosas desde el punto de vista presupuestario y sí que requieren del acuerdo y coordinación institucional. En este trabajo de identificación, análisis y planteamiento de soluciones de los "puntos de bloqueo" han participado numerosas asociaciones de usuarios ciclistas que han trasladado sus propuestas al CRTM.

\section{-Creación de aparcamientos seguros para bicicletas en estaciones de transporte público y en áreas de intercambio modal.}

Analizada la conectividad ciclista en un entorno urbano o metropolitano y mejorada la misma mediante la supresión de los llamados "puntos de bloqueo", es el momento de estudiar qué puntos de intercambio modal son los más adecuados para la instalación de aparcamientos de bicicletas. Se trata de ofrecer al ciclista un aparcamiento con unas condiciones mínimas de seguridad, en cuanto a su ubicación y al sistema de aparcabicis instalado. Esta Red de Estacionamientos de Bicicleta (REB) se está comenzando a implementar en el interior de la estaciones de Metro y Cercanías ofreciendo plazas de estacionamiento cubiertas y con seguridad "pasiva" al estar ubicadas en recintos video 
vigilados. Estos REBs están identificados con una imagen corporativa específica y se rigen por una normativa de uso pensada solo para ciclistas que acceden a la red de transporte. En una segunda fase, el REB se extenderá a áreas de intercambio modal con predominio del modo bus y con aparcabicis de exterior así como en estaciones de autobús comarcales o municipios de tamaño medio.

\section{-Normalización y reglamentación del marco legal que rige las condiciones de acceso de las bicicletas a las estaciones y vehículos de transporte público.}

Una de las medidas más demandadas por el colectivo ciclista en relación con el acceso al transporte público es la mejora de la normativa que rige el acceso de las bicicletas al interior de las instalaciones y a los vehículos de la red. En este sentido, se está haciendo un notable esfuerzo en la normalización y homogenización de los reglamentos/normativa que cada operador aplica con respecto al acceso de la bicicleta. Obviamente es imposible que exista una directriz común en este sentido ya que el modelo de explotación o de los vehículos no son similares entre operadoras y no tiene la misma capacidad para llevar bicicletas un tren, un metro o un autobús. En cualquier caso se está normalizando el reglamento para que sea lo más común posible a todas las operadoras y redes de autobús y también se estudia la mejora de las condiciones de acceso de la bicicleta al mayor operador de transporte de la región: Metro de Madrid.

-Desarrollos tecnológicos para integrar las tarjetas sin contacto de acceso a red de transporte público con las distintas tarjetas sin contacto de los sistemas de bicicleta pública favoreciendo el uso de la bici.

Esta es otra de las iniciativas en las que el CRTM está trabajando intensamente y con excelentes resultados. Desde 2015 todo el sistema de pago de los Abonos de Transporte del CRTM se lleva a cabo a través de tarjetas sin contacto, la llamada Tarjeta de Transporte Público (TTP). Lo que se ha logrado gracias al trabajo de los técnicos del CRTM y de los municipios implicados es hacer converger la tecnología de dicha TTP con la de las tarjetas de los sistemas de Bicicleta Pública. El primer municipio donde esto es ya una realidad es Getafe de tal manera que un viajero con una TTP del Consorcio puede liberar también con dicha tarjeta una bicicleta publica del municipio. A corto plazo, municipios como Leganés, Majadahonda o Rivas están en el proceso de adaptar el software de sus tarjetas para implementar también esta interesante medida de fomento de la intermodalidad.

\section{-Apoyo a cualquier iniciativa relacionada con la bicicleta y el transporte público incluidas también aquellas encaminadas a potenciar rutas cicloturistas o ciclo deportivas a las que se puede acceder en transporte público.}

Cualquier estudio que analice la interacción de bicicleta y transporte público no debe olvidar la importancia de las actividades de ocio en bicicleta como el ciclismo deportivo, el cicloturismo de carretera y las rutas en bicicleta de montaña. En este sentido, el territorio de la Comunidad de Madrid es un excelente botón de muestra del éxito de estas actividades, sobre todo en fin de semana. Lo paradójico del tema es que la mayoría de los desplazamientos que se realizan para montar en bicicleta se hacen en vehículo privado, cuando en realidad muchos de éstos se podrían hacer de manera sostenible llevando la bicicleta en transporte público. Este es el argumento de numerosos grupos ciclistas que hacen gala de realizar rutas con inicio y fin en una estación de Metro o Cercanías sin usar el automóvil. 
Con la nueva normativa que se está elaborando, para permitir el acceso de bicicletas a la red de autobuses interurbanos se abre un nuevo horizonte de rutas ciclistas por comarcas de la región, hasta ahora solo accesibles en vehículo privado. Esta apuesta tiene además una clara componente de apoyo al sector turístico y a iniciativas del propio Gobierno Regional como el proyecto Cicla Madrid una ruta circular para bicis de más de 400 kilómetros de longitud.

Por último, el CRTM desarrolla desde hace varios años una línea de trabajo de apoyo al ciclismo y excursionismo a pie mediante la edición de una colección de rutas desde estaciones de Metro y Cercanías. Esta colección descargable en formato PDF desde la Web del CRTM acaba de alcanzar la cifra de 16 itinerarios. Se trata de fomentar no solo las prácticas saludables sino también el conocimiento de espacios naturales urbanos y metropolitanos mediante el acceso a los mismos en Transporte Público.

\section{Una apuesta de futuro.}

El desarrollo por parte del Consorcio Regional de Transportes de Madrid de este programa de actuaciones de apoyo a la bicicleta y a su integración en el sistema de Transporte Público es una apuesta de futuro que, sin embargo, está teniendo unos excelentes resultados en el corto plazo ya que las operadoras de transporte y las administraciones implicadas en alguna de estas actuaciones están colaborando de un modo proactivo en su inminente aplicación con el convencimiento de que la bicicleta ha llegado a nuestras ciudades para quedarse y de que su papel en la intermodalidad ha de ser puesto en valor. 